\title{
Combinational ability of crossing components and heterosis of F1 hybrids of sugar sorghum by biomass yield
}

\author{
Oksana Kibalnik*, Dmitry Semin, Irina Efremova, and Tatiana Larina \\ FSBSI Russian Research and Design-Technological Institute of Sorghum and Corn, 1-y Institutskiy \\ Passage, 4, Saratov, Russia
}

\begin{abstract}
Currently, the most promising direction of breeding is the creation of F1 hybrids of sugar sorghum, based on the identification and selection of parental forms with high combinational ability. The source material and F1 hybrids were grown in the arid conditions of the Saratov Right Bank (Russia): the hydrothermal coefficient during the growing season was 0.68 . The evaluation of the combinational ability of crossing components was carried out using the topcross method. As a result of the tests, the L-52/13 line was identified with high GCA effects (15.71), and the highest SCA dispersions were found in the Saratovskoe 90 variety and the L-39/12 line (91.80-189.27). It was found that the type of sterile cytoplasm of iso-nuclear CMS-lines had a significant impact on the manifestation of SCA in terms of biomass yield: higher dispersion indicators were noted in 9E Zheltozernoye 10 (36.66). Genes with an additive effect are involved in the genetic control of this trait in paternal forms, and non-additive genes in maternal forms. In addition, each hybrid combinationwas observed with superdomination: the coefficient of phenotypic dominance varied from 2.01 to 18.91 . It is advisable to use the obtained information in the hybridization of sugar sorghum to obtain highheterosis hybrids.
\end{abstract}

\section{Introduction}

Currently, in many countries of the world, developments are underway to obtain an ecological fuel (for example, bioethanol), which would replace hydrocarbons [1]. Bioethanol production is the fastest growing industry in the world. It is mainly produced from easily reproducible raw materials: specially grown energy plants. These include sugar cane, sugar and grain sorghum, rice, sugar beet, potatoes, topinambur, corn, barley, rye and others. Nevertheless, for growing in arid regions, one of the most popular agricultural crops is sugar sorghum. This crop is characterized by a wide adaptive ability, high growth, content of water-soluble sugars in the juice of the main stem is up to 16-23\% [2]. At the same time, this plant has a high photosynthetic efficiency and can form a powerful biomass

\footnotetext{
*Corresponding author:kibalnik79@yandex.ru
} 
in a short time. Due to its biological characteristics, it is an alternative source of biofuels and sugar-containing products based on the processing of sugar sorghum stems [3-5]. Therefore, the creation of new highly productive varieties and hybrids of sorghum intended for use as an alternative raw material in the production of bioethanol is a promising direction in breeding.

It is known that F1 hybrids often exhibit the effect of heterosis for many economic characteristics, including biomass yield, which depends on correctly selected parental pairs and is widely used by breeders [6]. Therefore, the identification of crossing components with high combinational ability (CA) is the main stage in the breeding for heterosis, which also allows determining the inheritance of the studied trait, the participation of genes (additive and non-additive) in its control [7-8]. Usually, lines with cytoplasmic male sterility (CMS) are used as the maternal form, and varieties and lines are used as the paternal form. In addition, to expand the genetic diversity of first-generation hybrids, new types of sterile cytoplasms (A2, A3, A4 and others) were used in practical breeding along with the traditional A1 (milo) cytoplasm [9]. The influence of alternative types of sterile cytoplasms in sorghum has not been fully studied [10]. Therefore, the purpose of the research was to evaluate the combinational ability of CMS-lines based on sterile cytoplasms A3, A4, 9E in test crossings with samples of sugar sorghum; to identify heterotic F1 hybrids.

\section{Materials and methods}

Field studies were conducted at the experimental field of the Institute in 2018 (city of Saratov, Russia). The region is characterized by a sharply continental climate, a feature of which is the frequent manifestation of soil and air droughts during the growing season of agricultural crops. The hydrothermal coefficient for the period from the second decade of May to the second decade of September was 0.68 (the sum of active temperatures is $2696^{\circ} \mathrm{C}$, precipitation $-184.6 \mathrm{~mm}$ ), which indicates arid conditions. The soil of the experimental site is represented by southern medium-loamy chernozem. The humus content in the arable layer is $3.5 \%$. Nitrification capacity $-7.7 \mathrm{mg} / \mathrm{kg}$; phosphorus $-34.2-35.7$ $\mathrm{mg} / \mathrm{kg}$, potassium $-349-378 \mathrm{mg} / \mathrm{kg}$. The reaction of the soil medium $\mathrm{pH}=6.3-6.4$.

$F_{1}$ hybrids (39 in total) and parental forms were sown in a wide-row method with a row spacing of $70 \mathrm{~cm}$ in the third decade of May. The repetition in the experiment is threefold. The placement of plots with an area of $7.7 \mathrm{~m}^{2}$ is randomized. The plant stand was set manually -100 thousand $\mathrm{pl}$./ha. The accounting of the biomass yield was carried out according to the methodology of the state testing of agricultural crops [11]. The combining ability of the parental forms was determined by the topcross method [12].

True heterosis - heterosis over best parent $(\mathrm{H})$ and the coefficient of phenotypic dominance (hp) were determined by the formulas [13]:

$$
\begin{aligned}
& \mathrm{hp}=\left(\mathrm{F}_{1}-\mathrm{P}\right) /\left(\mathrm{P}_{\text {best }}-\mathrm{P}\right), \\
& \mathrm{H}=\left(\left(\mathrm{F}_{1}-\mathrm{P}_{\text {best }}\right) / \mathrm{P}_{\text {best }}\right) \times 100 \%,
\end{aligned}
$$

where $F_{1}$ is the value of the biomass yield in $F_{1}$ hybrids; $P$ is the average value of the trait in both parental forms; $\mathrm{P}_{\text {best }}$ is the value of the trait in the best parent form.

The varieties and lines of sugar sorghum (13 in total) used as a pollinator differed among themselves in the main economically valuable characteristics; whereas the CMSlines of grain sorghum (3 in total) A3 Zheltozernoye 10, A4 Zheltozernoye10 and 9E Zheltozernoye 10 differed only in the type of sterile cytoplasm. 
Statistical processing of experimental research results was performed by a one-factor analysis of variance using the program "Agros 2.09".

\section{Results and discussion}

The variance analysis confirmed the differences between the tested varieties of sugar sorghum and F1 hybrids in terms of biomass yield. Of the paternal forms of hybrids, the highest productivity was characterized by L-52/13 (28.05 t/ha), k-64 (29.27 t/ha) and L42/13 (30.55 t/ha) (Fig. 1). In hybrids, the biomass yield varied from $30.7 \mathrm{t} / \mathrm{ha}$ (in crossings with the Kamyshinskoe 8 variety) to 71.4 t/ha (in crossings with the L-52/13 line) (Fig. 2).

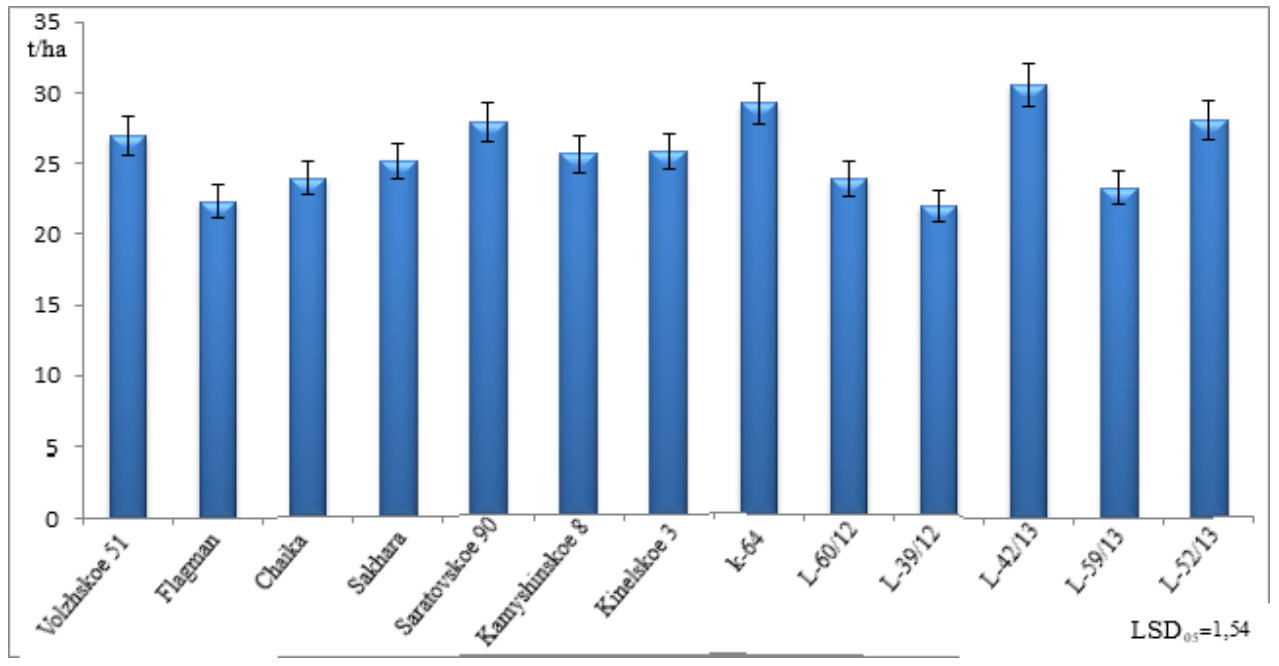

Fig. 1. Yield of biomass of sugar sorghum cultivars, $\mathrm{t} / \mathrm{ha}$.

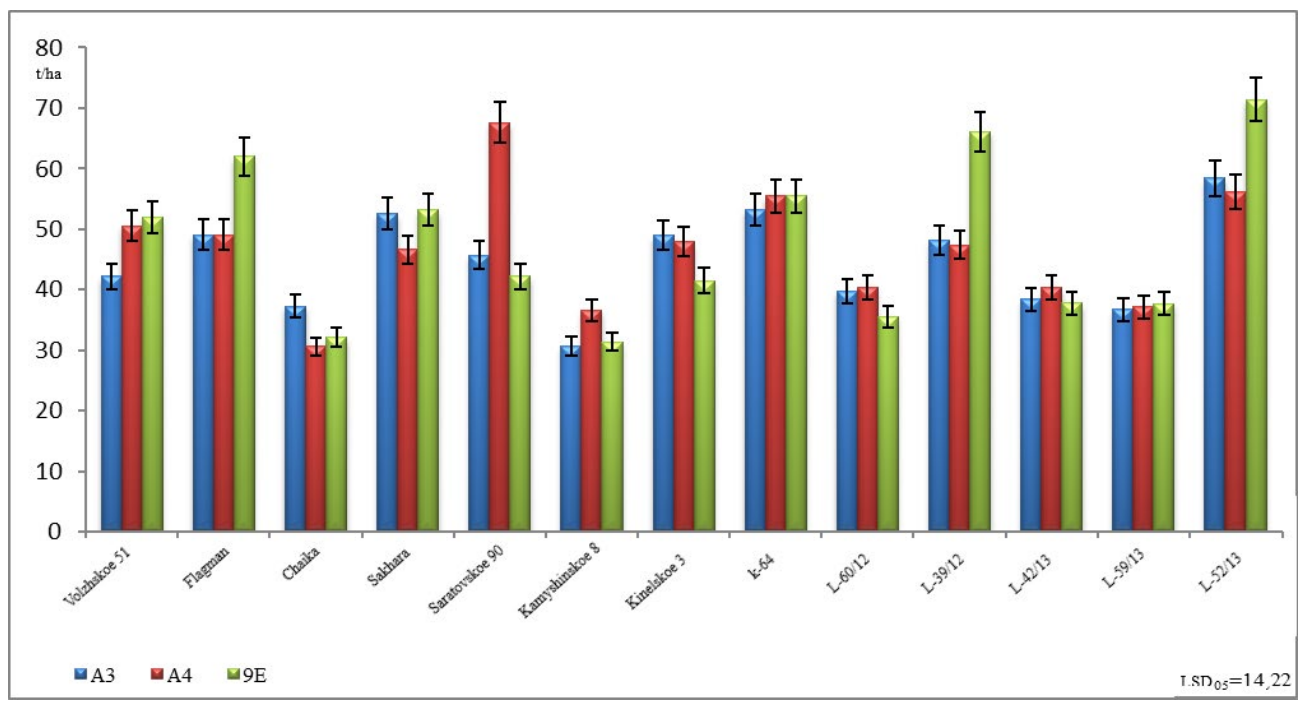

Fig. 2. Biomass yield of F1 hybrids based on iso-nuclear CMS-lines with cytoplasm types A3, A4 and 9E, t/ha. 
The assessment of the CA of sorghum sugar variety samples showed that the Kamyshinskoe 8 (-13.39) and Chaika (-12.96) varieties are characterized by a low general combinational ability (GCA), and the L-52/13 line (15.71) has the greatest GCA effects. Samples with an average GCA were also identified. Such paternal forms should include Sakhara, Saratov 90, Flagman, L-39/12 and k-64. The positive effects of the GCA of these pollinators varied in the range of 4.48-8.48 (Table 1). A high specific combination ability (SCA) was established in the Saratovskoe 90 variety and the L-39/12 line, as evidenced by the highest dispersion indicators of SCA - 91.80-189.27 (Table 1). The L-52/13 line and the Flagman variety distinguished themselves by the average values of SCA variances (41.17-53.74). It should be noted that the breeding lines of sugar sorghum of Institute of Sorghum and Cornshowed a high combinational ability in crosses based on CMS-lines with the type of sterile cytoplasm A2 - A2 O-1237, A2 AGS, A2 KVV 114 [7]. The ratio of standard deviations of the general and specific combinational ability for biomass yield indicates that genes with an additive effect participate in genetic control in paternal forms $\left(\mathrm{ms}_{\mathrm{GCA}} / \mathrm{msSA}_{\mathrm{SCA}}=6.31\right)$.

Table 1. Combinational ability of sugar sorghum samples for biomass yield.

\begin{tabular}{|c|c|c|c|c|c|}
\hline \multirow{2}{*}{ Sample } & \multirow{2}{*}{ GCA effects } & \multicolumn{3}{|c|}{ SCA effects } & \multirow{2}{*}{ SCA variance } \\
\hline & & A3 & A4 & 9E & \\
\hline Volzhskoe 51 & 1.91 & -4.42 & 1.99 & 2.43 & 14.69 \\
\hline Flagman & 7.11 & -2.72 & -4.61 & 7.33 & 41.17 \\
\hline Chaika & -12.96 & 5.55 & -3.04 & -2.51 & 23.16 \\
\hline Sakhara & 4.48 & 3.32 & -4.48 & 1.16 & 16.19 \\
\hline Saratovskoye 90 & 5.54 & -4.55 & 15.46 & -10.91 & 189.27 \\
\hline Kamyshinskoye 8 & -13.39 & -0.62 & 3.39 & -2.77 & 9.78 \\
\hline Kinelskoye 3 & -0.16 & 4.45 & 1.46 & -5.91 & 28.39 \\
\hline K-64 & 8.48 & 0.12 & 0.42 & -0.54 & 0.24 \\
\hline $\mathrm{L}-60 / 12$ & -7.72 & 2.82 & 1.52 & -4.34 & 14.53 \\
\hline L-39/12 & 7.58 & -4.19 & -6.78 & 10.96 & 91.80 \\
\hline $\mathrm{L}-42 / 13$ & -7.46 & 1.15 & 1.16 & -2.31 & 3.99 \\
\hline L-59/13 & -9.12 & 1.12 & -0.38 & -0.74 & 0.97 \\
\hline $\mathrm{L}-52 / 13$ & 15.71 & -2.02 & -6.11 & 8.13 & 53.74 \\
\hline F fact. & $11.68^{*}$ & & $1.85^{*}$ & & $1.85^{*}$ \\
\hline $\mathrm{ms}$ & 256.68 & & & & 40.66 \\
\hline
\end{tabular}

Note: $* \mathrm{p} \leq 0.05$.

Analysis of the GCA effects of hybrids allowed to identify promising combinations of crossings. Thus, hybrids 9E Zheltozernoye 10/Flagman (7.33), 9E Zheltozernoye 10/L52/13 (8.13), 9E Zheltozernoye 10/L-39/12 (10.96) and A4 Zheltozernoye 10/Saratovskoye 90 (15.46) were characterized by the highest values.

With the correct selection of crossing components, important attention is paid to the choice of the maternal form. The influence of sterile cytoplasms on the combinational ability of iso-nuclear CMS-lines for breeding-valuable traits in sorghum is not only of fundamental, but also of practical interest: for example, the absence of alternative types of sterile cytoplasms can lead to the termination of the production of hybrids in case of mass lesions of their diseases or pests. In our studies, the type of sterile cytoplasm did not have a significant effect on the GCA of sterile lines in terms of biomass yield $\left(\mathrm{F}_{\text {fact }}<\mathrm{F}_{\text {teor }}\right)$. Nevertheless, higher GCA effects were noted in the CMS-line 9E Zheltozernoye $10-1.27$. Significant differences were found in terms of SCA dispersion: the highest values were also found in the maternal line on the cytoplasm 9E - 36.66 (Fig. 3). The ratio of standard 
deviations showed that the maternal forms have an advantage of non-additive effects over additive ones in the genetic control of this trait: $\mathrm{ms}_{\mathrm{GCA}} / \mathrm{ms}_{\mathrm{SCA}}=0.67$.
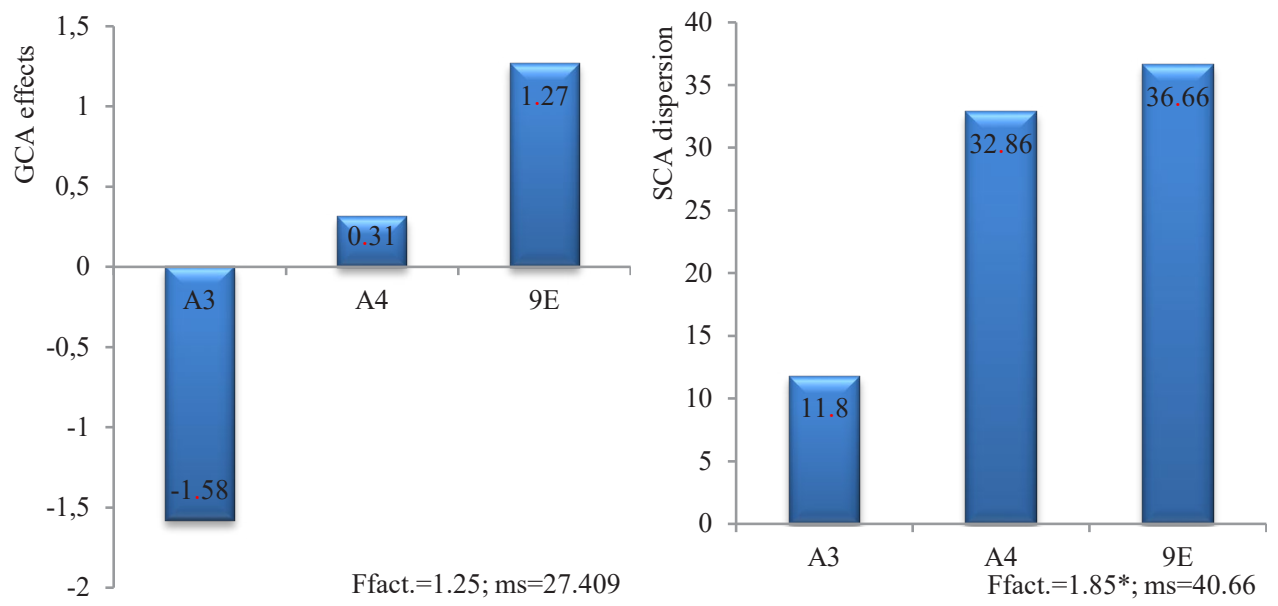

Note: $* \mathrm{p} \leq 0.05$.

Fig. 3. GCA effects and SCA dispersion of sorghum iso-nuclear CMS-lines based on sterile cytoplasms A3, A4 and 9E

The results obtained on the absence of a cytoplasmic effect on the combinational ability of iso-nuclear CMS-lines with different types of sterile cytoplasms in terms of biomass yield in crossings with samples of sugar sorghum are reflected in the publication of Hoffmann and Rooney [14]. At the same time, in crossings with samples of grain sorghum, a significant influence of cytoplasm 9E was noted, especially manifested in dry seasons [15].

F1 hybrids created on the basis of iso-nuclear CMS-lines (A3, A4 and 9E Zheltozernoye 10) and samples of sugar sorghum showed a different degree of true heterosis in terms of biomass yield from $19.7 \%$ to $200.0 \%$. Moreover, 12 out of 39 hybrids have an advantage over the best parental form of more than $100 \%$. The hybrids obtained during hybridization of the CMS-line 9E Zheltozernoye 10 with the samples Flagman, L-39/12, L-52/13 (154.5$200.0 \%$ ) were distinguished by the greatest true heterosis.

The determination of phenotypic dominance coefficients in the yield of biomass of F1 hybrids showed an overdominance of biomass yield, which is especially pronounced in combinations of crossings of CMS-line 9E Zheltozernoye 10 with the Flagman - 16.20 variety and the L-39/12 - 18.91 (Table 2).

Table 2. True heterosis (\%) and the coefficient of phenotypic dominance of F1 hybrids based on the crossing of iso-nuclear CMS lines with the types A3, A4 and 9E of cytoplasms in terms of biomass yield.

\begin{tabular}{|l|c|c|c|c|c|c|}
\hline \multirow{2}{*}{ Sample } & \multicolumn{3}{|c|}{ Heterosis (\%) } & \multicolumn{3}{c|}{ Coefficient of phenotypic dominance } \\
\cline { 2 - 7 } & A3 & A4 & 9E & A3 & A4 & 9E \\
\hline Volzhskoe 51 & 56.7 & 87.5 & 92.7 & 3.70 & 4.99 & 6.09 \\
\hline Flagman & 120.0 & 120.0 & 177.8 & 8.97 & 8.42 & 16.20 \\
\hline Chaika & 55.8 & 27.8 & 34.1 & 4.20 & 2.51 & 3.39 \\
\hline Sakhara & 108.7 & 85.2 & 111.4 & 6.72 & 5.26 & 7.96 \\
\hline Saratovskoye 90 & 63.6 & 142.0 & 51.1 & 3.88 & 7.19 & 3.64 \\
\hline Kamyshinskoye 8 & 19.7 & 42.7 & 22.4 & 2.01 & 3.08 & 2.35 \\
\hline
\end{tabular}




\begin{tabular}{|l|c|c|c|c|c|c|}
\hline Kinelskoye 3 & 89.6 & 85.3 & 60.5 & 5.52 & 5.11 & 4.58 \\
\hline K-64 & 82.1 & 89.6 & 89.6 & 4.52 & 4.70 & 5.31 \\
\hline L-60/12 & 66.4 & 68.9 & 48.4 & 4.82 & 4.74 & 4.40 \\
\hline L-39/12 & 118.3 & 115.2 & 200.0 & 9.12 & 8.33 & 18.91 \\
\hline L-42/13 & 25.7 & 31.9 & 23.7 & 2.05 & 2.26 & 2.08 \\
\hline L-59/13 & 57.7 & 59.4 & 62.0 & 4.51 & 4.39 & 5.69 \\
\hline L-52/13 & 108.2 & 100.4 & 154.5 & 5.88 & 5.35 & 8.93 \\
\hline
\end{tabular}

\section{Conclusions}

The analysis of the combinational ability of the parent forms of grain and sugar sorghum allowed to identify components with high GCA and SCA. It was found that in these test crossings, the genetic control of the biomass yield of sugar sorghum cultivars is controlled by genes with an additive effect, and the non-additive isonuclear CMS-lines of grain sorghum, as evidenced by the values of the ratio of standard deviations -6.31 and 0.67 , respectively. Highly productive F1 hybrids are recommended to be created with the involvement of the Flagman and Saratovskoe 90 varieties, the L-39/12 and L-52/13 lines as the male parent. It should be noted that the greatest practical significance in crosses with samples of sugar sorghum was noted in the CMS-line based on cytoplasm 9E, characterized by a high dispersion of SCA on this trait. The greatest true heterosis was also manifested in F1 hybrids using 9E Zheltozernoye 10. Based on the literature data and the experimental results obtained by us, it should be assumed that the manifestation of cytoplasmic effects in sorghum hybrids depends on the hydrothermal factors of the season in the cultivation region, as well as on the specific interaction of the genotypes of the crossing components, which must be considered in further breeding work to create highly productive sugar sorghum hybrids.

\section{Acknowledgment}

The work was carried out within the framework of the state task of the Ministry of Agriculture of the Russian Federation and the thematic plan of the Russian Research and Design and Technological Institute of Sorghum and Corn.

\section{References}

1. A.V. Umakanth, A.A. Kumar, V.A. Tonapi,Sweet Sorghum for Biofuel Industry. Breeding Sorghum for Diverse End Uses, 255 (Elsevier Ltd., 2018)

2. C. Olweny, G. Abayo, M.M. Dida, P. Okori, Sugar Tech 19(1), 57 (2017)

3. M.J. da Rocha, J.A.R. Nunes, R.A. da Costa Parrella, P.S. da silva Leite, G.M.R. Lombardi, M.L.Costa Moura, R.E.Schaffert, A.T.Bruzi, CBAB (18), 365 (2018)

4. F. Jia, J. Chawhuamak, M.R. Riley, W. Zimmt, K.L. Ogden. J. of Biol. Engineering 7, 1 (2013)

5. M.J. da Silva, C.M.B. Damasceno. J.E. de Souza Carneiro, H.D. Pereira, P.C. Souza Carneiro, R.E.Schaffert, R.A. da Costa Parrella, Agronomy J. 112, 1549 (2020)

6. S.He, C. Tang, M.L. Wang, S. Li, B. Diallo, Yi Xu, F. Zhou, L. Sun, W. Shi, G.H. Xie. Industrial Crop\&Products (157), 112894 (2020) 
7. V.I. Zhuzhukin, V.S. Gorbunov, O.P. Kibalnik, D.S. Semin, A.YuGarshin. Russian Agricultural Sciences 43(6), 456 (2017)

8. I.C.M. Oliveira, T.d.S. Marçal, K. da Costa Bernardino, P.C.d.O. Ribeiro, R.A. da Costa Parrella, P.C.S. Carneiro, R.E. Schaffert, J.E.d.S. Carneiro. Crop Sci. (59), 1554 (2019)

9. B.V.S. Reddy, S. Ramesh, P.S. Reddy, A.A. Kumar, Indian J.Genet69(3), 199 (2009)

10. O.P. Kibalnik, L.A. Elkonin. Vavilov J. of genetics and Breeding 24(6), 549 (2020)

11. Methods of state variety testing of agricultural crops, 2, 194 (1989)

12. V.K. Savchenko,Method for evaluating the combining ability of genetically different sets of parental forms. Methods of genetic-breeding and genetic experiments, 48 (1973)

13. Y.L. Guzhov, A. Fuks, P. Valichek,Selection, and Seed Farming of the Cultivated Plants, 536 (1999)

14. L. Hoffmann, W.L. Rooney. J. of Sustainable Bioenergy Systems,2, 129 (2013)

15. L. Elkonin, O. Kibalnik, A. Zavalishina, G. Gerashchenkov, Genetic Function of Cytoplasm in Plants with Special Emphasis on Sorghum, In Chloroplasts and Cytoplasm. Structure and Function (eds. C. Dejesus, L. Trask), 97 (2018) 